$\mathrm{Li}, \mathrm{X}$.Z., et al., A Chinese View on Summary of Condensed Matter Nuclear Science. J. Fusion Energy, 2004. 23(3): p. 217-221.

The original publication is available at www.springerlink.com

\title{
A Chinese View on Summary of Condensed Matter Nuclear Science
}

\author{
Xing Z. Li, Bin Liu, Qing M. Wei, Shu X. Zheng ${ }^{1}$, Dong X. Cao ${ }^{1}$ \\ Department of Physics, Tsinghua University, Beijing 100084, CHINA \\ ${ }^{1}$ Department of Engineering Physics, Tsinghua University, Beijing 100084, CHINA \\ Lxz-dmp@tsinghua.edu.cn
}

Key words: excess tritium; selective resonant tunneling model; deuterium flux;

condensed matter nuclear science; cold fusion.

\begin{abstract}
Investigation on tritium was one of the recommendations in ERAB report of DOE in November, 1989. 15 year evolution of the related research proved that it was an important recommendation. A selective resonant tunneling model is attempted to explain this discovery. Deuterium flux might be a key issue to solve the problem of the reproducibility. A further investigation is suggested based on this model.
\end{abstract}

\section{EXCESS TRITIUM}

November, 1989 ERAB made five recommendations. "Investigations designed to check the reported observations of excess tritium in electrolytic cells are desirable.” was one of them ${ }^{[1]}$. It was important, because the existence of tritium product should be a critical evidence to confirm the nuclear reactions in the electrolytic cells ${ }^{[2]}$. On the other 
hand, the quantity of the tritium was incommensurate with the neutron emission. It should be a hint that the origin of this tritium should be different from the usual $d+d$ fusion reaction. Particularly, there was not any report on $14 \mathrm{MeV}$ neutron emission. It should be another hint that this tritium should not be the product of $d+d$ fusion reaction.

F. Will conducted a series of carefully designed experiments which showed that excess tritium in electrolytic cells and gas-discharge tube were real and reproducible ${ }^{[3]}$. In parallel with his gas discharge experiments, T. Claytor of Los Alamos National Lab. showed also that the excess tritium in gas-loading systems was real as well ${ }^{[4]}$. This tritium measurement was confirmed by the mass spectroscopy in $2001^{[5]}$. Indeed tritium appeared in various experiments in Japan, Italy, Russia, US, Canada, India and China. It is one of the strongest evidences for condensed matter nuclear science in the past 15 years. It implied a new mechanism at low energy for nuclear reaction.

\section{SELECTIVE RESONANT TUNNELING MODEL}

Selective resonant tunneling model has been proposed to provide the mechanism for this low energy nuclear reaction in the condensed matter. Before we hop into the issue of tritium production, we may introduce the basic concept of the selective resonant tunneling model first.

Selectivity of the resonant tunneling can be learnt from the electronic harmonic circuit. A harmonic circuit is able to pick up the specific wave frequency from the air. When the signal is weak, it requires low resistance of circuit in order to observe this resonance before its damping off. Similar situation is expected in resonant tunneling of the Coulomb barrier. At the low energy, the Coulomb barrier is thick and high; hence, 
the incident deuteron wave in the nuclear well is very weak due to the Coulomb barrier. The amplitude of weak penetrating wave may be enhanced by the resonance effect when the phase of the reflected wave inside the nuclear well is same as that of the incident wave. This is the resonant tunneling. This resonant tunneling requires a weak damping in order to observe this resonance before its damping off also. The damping in resonant tunneling of the Coulomb barrier is just the fusion reaction itself, because the deuteron wave function disappears due to the fusion reaction. Thus, this fusion reaction rate cannot be very fast, otherwise the fast damping will kill the resonant effect. On contrary, this fusion reaction rate cannot be very small, because zero reaction rate would not give any fusion reaction even if the incident deuteron wave function has a large amplitude inside the nuclear well due to the resonance. As a result, the life-time of the deuteron wave function cannot be too large or too small. There is an optimized life-time, $\tau_{\text {life }}$, to match a specific Coulomb barrier ${ }^{[6]}$ :

$$
\tau_{\text {life }} \approx \theta \mid \tau_{\text {flight }}
$$

Here, $\theta$ is a very large number for a thick and high Coulomb barrier. ( $\theta$ is in the order of $10^{22} \sim 10^{31}$ or greater here. $1 / \theta^{2}$ is just the famous Gamow penetration factor); $\tau_{\text {flight }}$ is the flight-time inside the nuclear well for the penetrating deuteron. $\tau_{\text {flight }}$ is of the order of $10^{-23}$ seconds.

Now we may understand why there is no neutron emission from the resonant tunneling at low energy, because the life-time for a neutron emission process is too short to meet the matching condition (1). (Usually, the life-time for a neutron emission process is of the order of $10^{-23}$ seconds due to the nature of the "Strong Nuclear 
Interaction” between nucleons.) Electromagnetic interaction is much weaker than the strong nuclear interaction (the life-time for a gamma-ray emission process here is of the order of $10^{-17}$ seconds), but it is still too strong to meet the matching condition $(1)^{[7,8]}$. Only the weak interactions ( $\beta$-decay or K-capture) might possibly provide the life-time to meet this matching condition (1) (Fig.1).

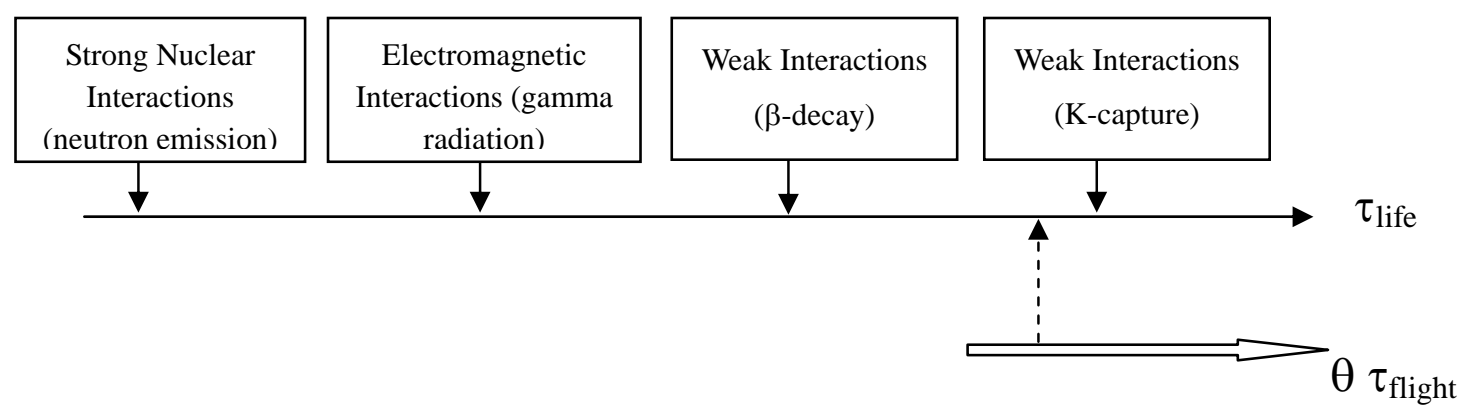

Fig.1 Schematics showing the selectivity of resonant tunneling model

Consequently, this selective resonant tunneling answers three famous questions proposed by J. R. Huizenga in $1992{ }^{[9]}$ : the resonant tunneling provides the mechanism to penetrate the Coulomb barrier; and the selectivity of the resonant tunneling at low energy explains why there is no neutron or gamma radiations after the resonant tunneling at low energy. It is clear now that neutron detection is not a good choice to search for this low energy resonance. The Nobel prize winner, Professor B.D. Josephson of Cambridge University, cited twice our work on "selective resonant tunneling model” in his recent talk at Lindau Meeting ${ }^{[10]}$ in order to reply to the "pathological disbeliever”. 
It is interesting to find that this selective resonant tunneling is supported by the hot fusion data as well ${ }^{[11]}$. Indeed this selective resonant tunneling model corrected a mistake in the $\mathrm{d}+\mathrm{t}$ fusion cross section data provided by National Nuclear Data Center (Brookhaven) in $1996^{[12]}$. Recently this model has been successfully extended to the calculation of the hot fusion cross-section for $\mathrm{d}+\mathrm{d}$, and $\mathrm{d}+{ }^{3} \mathrm{He}{ }^{[13]}$. This encouraged us to apply this model to study the puzzle of tritium production.

\section{THE WEAK INTERACTION FOR TRITIUM PRODUCTION}

If the weak interaction is the only possible reaction for the resonant tunneling at low energy; then, we may immediately write down two reactions for tritium production in $\mathrm{D}(\mathrm{H}) / \mathrm{Pd}$ systems, i.e.

$$
\begin{aligned}
& p+d \rightarrow T+e^{+}+v_{e} \\
& p+d \stackrel{\text { K-capture }}{\longrightarrow} T+v_{e}
\end{aligned}
$$

Usually, the positron-decay is faster than the K-capture process for a parent nucleus; however, in case of the resonant tunneling, this positron-decay (2) is too fast to meet the matching condition (1). Only the K-capture process (3) would possibly match the selective resonant condition (1). This inference has been consistent with the experiments. The annihilation of positron would produce the $0.511 \mathrm{MeV}$ gamma radiation. However, we did not observe any commensurate gamma radiation in all these tritium production experiments. Indeed the light water was detected in all Bockris experiments which produced tritium ${ }^{[14]}$, and T. Claytor reported that slight tritium production was observed in his control run with hydrogen as well ${ }^{[15]}$. It simply meant that proton was in favor of 
tritium production. The hydrophilic nature of the heavy water might explain the contamination by the light water in the electrolytic cells. Moreover, triton in reaction (3) obtains the recoil energy only, which is in the order of keV; hence, there was no $14 \mathrm{MeV}$ neutrons observed in any of these tritium production experiments.

It is interesting to notice that $\mathrm{H}$. A. Bethe, the Nobel prize winner, used weak interaction and resonant tunneling to obtain the right answer for the $\mathrm{p}^{+} \mathrm{p}$ fusion cross section in the sun ${ }^{[16]}$ as well.

\section{THE ROLE OF THE CONDENSED MATTER: ION BAND}

The condensed matter is important for this resonant tunneling to be in effect. At low energy, the long life-time state in the resonant tunneling corresponds to a very narrow energy level. It cannot be realized through the beam-target experiment using accelerator because of the dispersiveness of the beam energy. Even if in a solid, the confined deuteron state is oscillatory in energy due to the thermal oscillation of the crystal lattice. In a cycle of oscillation, only in an extreme small portion of oscillation, a deuteron might be in a resonant tunneling state. We need an energy band ${ }^{[17]}$ for the deuterons (or protons) in a lattice and let a number of deuterons to fill in this energy band. Whenever this energy band is overlapped with this resonant tunneling state, one of these deuterons in the energy band is always in the resonant tunneling state. Condensed matter provides the necessary periodicity to form this energy band. Certain metals (Pd, $\mathrm{Ni}$, $\mathrm{Ti}$, etc.) are particularly good because of their nature in absorbing hydrogen, which means an almost full occupation of this energy band. In the first five years of the cold fusion research, a lot of efforts were devoted to load deuterium gas into the palladium in order to solve the 
problem of reproducibility.

\section{REPRODUCIBILITY AND THE DEUTERIUM FLUX}

Gradually, it was realized later in a variety of the experiments that deuterium flux played an important role in repeating the experiment ${ }^{[18][19][20]}$. The concept of energy band for deuterons simply means that the deuterons inside a crystal might be described by a single wave function. This concept has further led to an anomalous deuterium flux on the crystal surface. The reflected waves from the different surface layers of the crystal might interfere destructively with each other, and the total reflection might be greatly reduced. The result is the enhancement of deuteron density in the surface layers, because the penetration on the surface is greatly enhanced due to the reduction of the reflection. This inference has been supported by the experimental results that most of the nuclear reactions appear in the surface layers ${ }^{[19]}$. Moreover, multiple layer coating has been applied to further enhance this effect; since then, the reproducibility is improved in various laboratories. We no longer pursue the high loading ratio inside the bulk of the palladium, instead, we attempt to increase the local deuteron density in the surface thin layer in terms of high deuterium flux. The key issue is the rapid change of the gradient of deuteron density which maintains a steady state of self-sustaining resonant tunneling reaction region. Fig. 2 shows that a heat flow of $2 \mathrm{~mW}$ was maintained in 2500 seconds while a deuterium flux was permeating through the thin wall of a palladium tube. The right hand side of Fig.2 shows the schematics of the apparatus. Deuterium gas was fed into a thin palladium tube which was installed in the center of a reaction chamber. When the temperature of the Pd tube and the reaction chamber increased, there was a 
deuterium flux permeating through the thin Pd wall. The deuterium flux was pumped out through a coaxial stainless steel tube. The reaction chamber sat in a high-precision calorimeter (Setaram C-80D) to monitor the heater flow. A heat flow was observed when the whole system was cooling down slowly from $150^{\circ} \mathrm{C}$ to $140^{\circ} \mathrm{C}$ (left-lower plot of figure 2). The left-upper plot of figure 2 shows the heat flow (dot-dash-dot line) in parallel with the deuterium flux (solid line). This deuterium flux increased first before it decreased with temperature. This is very different from the feature of a normal diffusion flux which is supposed to decrease monotonically with the decreasing temperature. We may call it anomalous deuterium flux which was correlated with a heat flow. It is reproducible.

\section{SUGGESTION FOR FURTHER EXPERIMENTS}

The heat carried by the anomalous flux is more than $120 \mathrm{eV}$ per deuteron which is more than any conceivable chemical heat. Hence, it is desirable to conduct the mass spectroscopy analysis for the permeating gas. Particularly, if tritium is observed in the permeating gas when a mixture of $\mathrm{H}_{2}$ and $\mathrm{D}_{2}$ gas are used to feed the Pd tube. It would justify not only the nuclear nature of the heat flow, but also the "selective resonant tunneling theory”. The early Bockris’ electrolytic cell ${ }^{[14]}$ and Claytor's hydrogen cycle [15] might be two precedent experimental results that support this proposal. The preliminary experiments at Energy Conversion Research Laboratory (HEAT project proposed by Dr. Glenn Schmidt at New Mexico Tech.) and at Institute of Plasma Physics (Hefei) were encouraging ${ }^{[21]}$. 

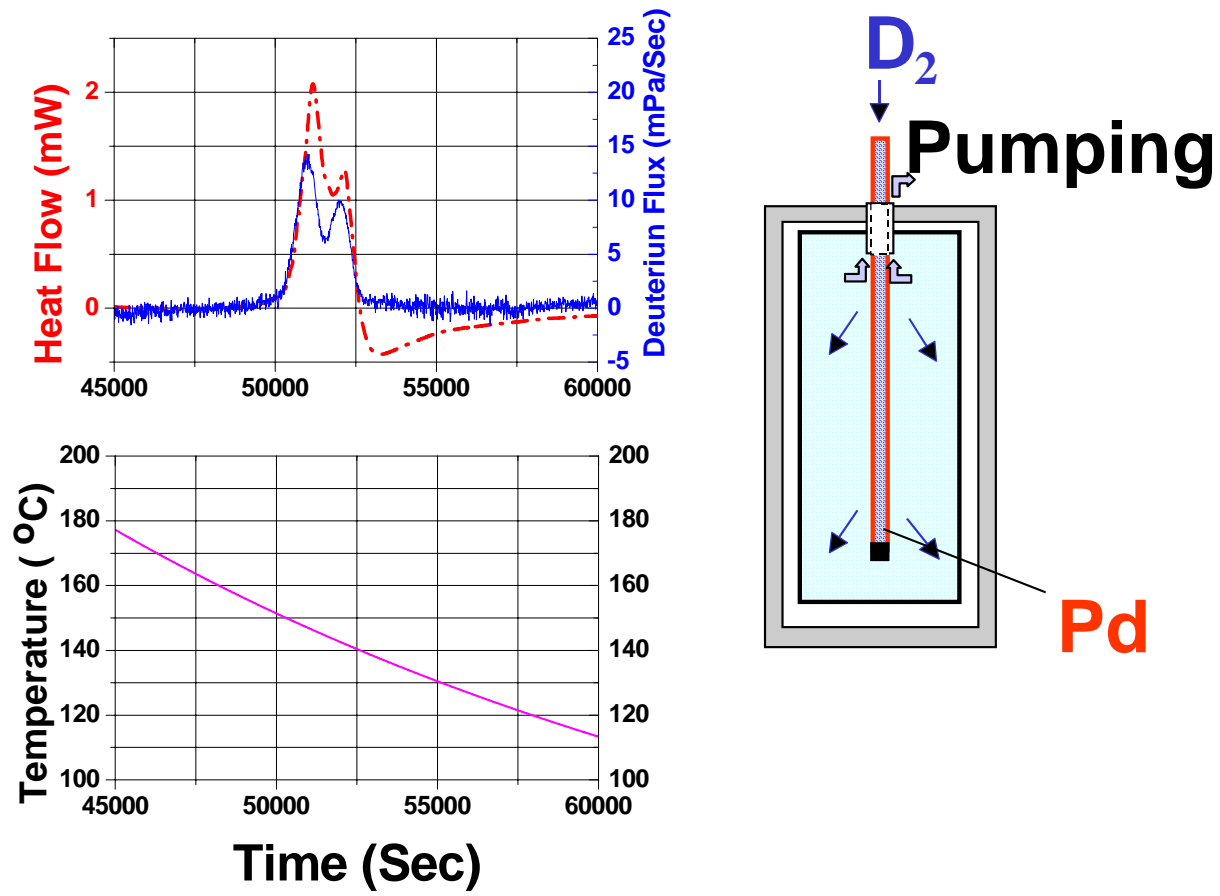

Fig.2 A heat flow correlated with an anomalous deuterium flux permeating through the thin wall of a Pd tube cooling down to certain temperature interval.

\section{CONCLUDING REMARK}

(1) Resonance plays key role in the Condensed Matter Nuclear Science. Indeed, there is not any violation of the law of the physics.

(2) It is not easy to keep this resonance in a steady state.

(3) We are supposed to look for the mechanism which may keep this resonant state in a self-sustaining way. Possibly, a deuterium flux permeating through Pd film is a method to maintain this resonance.

(4) Fission was discovered in 1939, the first fission reactor was in operation in 1942 with the support from the whole nation. "Cold fusion” was announced in 1989, but we are still in the stage of confirmation of this phenomenon because of the lack of financial support. 


\section{ACKNOWLEDGEMENTS}

This work is supported by The Ministry of Science and Technology (Fundamental

Division), Natural Science Foundation of China (\#10145004, and \#10475045) and Tsinghua University (Basic Research Fund, 985-II). We are grateful to Physics Section of IAEA for its continuous support.

\section{REFERENCES}

\section{$\underline{\text { Excess Tritium }}$}

[1] Energy Research Advisory Board, Cold Fusion Research, DOE/S-0073, 1989.

[2] N.J.C. Packham, K.L. Wolf, J.C. Wass, R.C. Kainthla, and J. O’M. Bockris, "Production of Tritium from $\mathrm{D}_{2} \mathrm{O}$ Electrolysis at a Palladium Cathode," J. Electroanal. Chem., 270 415(1989).

[3] F. G. Will, K. Cedzynska, D.C. Linton, "Reproducible Tritium Generation in Electrolychemical Cells Employing Palladium Cathodes with High Deuterium Loading,” J. Electroanal. Chem., 360161 (1993).

[4] T. N. Claytor, et al., “Tritium Production from Palladium Alloys,” Proceedings of ICCF-7, Vancouver, Canada, ENECO, Inc., Salt Lake City, USA (1998) p.88.

[5] B.W. Clarke, B.M. Oliver, M.C.H. Mckubre, F.L. Tanzella, P. Tripodi, "Search for ${ }^{3} \mathrm{He},{ }^{4} \mathrm{He}$ in Arata-Style Palladium Cathodes II: Evidence for Tritium Production,” Fusion Science and Technology, 40152 (2001).

\section{Selective Resonant Tunneling model}

[6] X.Z. Li, C.X. Li, and H.F. Huang, "Maximum Value of the Resonant Tunneling Current through the Coulomb Barrier,” Fusion Technology 36324 (1999). 
[7] X. Z. Li, J. Tian, and M. Y. Mei "Fusion Energy without Strong Nuclear Radiation," Journal of Fusion Energy, 18, 51 (1999).

[8] X. Z. Li, et al., "Study Of Nuclear Physics For Nuclear Fusion," Journal of Fusion Energy, 19, 163 (2002).

\section{Three Miracles}

[9] J. R. Huizenga, “Cold Fusion: The Scientific Fiasco of the Century,” University of Rochester Press, p.111-113 (1992).

[10] B. D. Josephson, “Pathological Disbelief”, Lecture given at the Nobel Laureates’ Meeting, Lindau, June 30th., 2004, Edited version of presentation (revised Aug. 20th., 2004, http://www.newenergytimes.com/library/2004JosephsonB-LindauLecture.pdf )

\section{Selective Resonant Tunneling model and Hot Fusion}

[11] X.Z. Li, J. Tian, M.Y. Mei, and C.X. Li, "Sub-barrier Fusion and Selective Resonant Tunneling,” Phys. Rev. C, 61024610 (2000).

[12] X.Z. Li, “Nuclear Physics for Nuclear Fusion,” Fusion Science and Technology, 41 63 (2002).

[13] X.Z. Li, B. Liu, S. Chen, Q. M. Wei, H. Hora, “Fusion cross sections for inertial fusion energy,” Laser and Particle Beams, 22 (2004), 469.

Hydrogen in experiments producing tritium

[14] K. L. Wolf, “Wolf: My Tritium was an Impurity,” Science, 2481301 (1990).

[15] T. N. Claytor, et al., “Tritium Production from Palladium Alloys,” Proceedings of ICCF-7, Vancouver, Canada, ENECO, Inc., Salt Lake City, US (1998) p.88.

$\underline{\text { Resonant Tunneling and Weak Interaction in the Sun }}$ 
[16] H.A. Bethe, C. L. Chritchfield, "The Formation of Deuterons by Proton Combination,” Phys. Rev. 54246 (1938).

$\underline{\text { Ion Band in Crystal }}$

[17] T.A. Chubb and S.R. Chubb, "Cold Fusion as an Interaction between Ion Band States,” Fusion Technology, 2093 (1991).

Deuterium Flux and Reproducibility

[18] M.C.H. Mckubre, S. Crouch-baker, A.K. Hauser,S.I. Smedley, F.L. Tanzella, M.S. Williams, S.S. Wing, “Concerning Reproducibility of Excess Heat Production,” Proceedings of ICCF-5, Monte-Carlo, Monaco (1995) p.17.

[19] Y. Iwamura, M. Sakano, and T. Itoh, “Elemental Analysis of Pd Complexes: Effects of $D_{2}$ Gas Permeation,” Jpn. J. Appl. Phys. 414642 (2002).

[20] X.Z. Li, B. Liu, J. Tian, Q.M. Wei, R. Zhou, and Z.W. Yu, “Correlation between Abnormal Deuterium Flux and Heat Flow in a D/Pd System,” J. Phys. D: Appl. Phys. 363095 (2003).

[21] Q. M. Wei, B. Liu, Y. X. Mo, X. Z. Li, S. X. Zheng, D. X. Cao, X. M. Wang, and J. Tian, “Deuterium (Hydrogen) Flux Permeating through Palladium and Condensed Matter Nuclear Science”, The Proceedings of 12-th International Conference on Condensed Matter Nuclear Science, Marseilles, France, Oct.31-Nov.5, 2004. edited by Jean-Paul Biberian.(to be published) 